\title{
Comportamiento de la atipia epitelial cervical en seguimiento a mediano plazo. Un estudio comparativo
}

\author{
Myriam Bravo de I*; Ligia Castro de O.**; Braulio Lara***
}

\begin{abstract}
RESUMEN cervical.

OBJETIVO: Determinar el riesgo para desarrollar Neoplasia intraepitelial o cáncer invasor en pacientes con atipia epitelial en frotis

METODO: Este estudio comparativo hace seguimiento en un tiempo promedio de 36 meses a 135 pacientes, organizadas en 2 grupos. El primero, formado por 52 mujeres con diagnóstico de atipia epitelial de significado indeterminado (atipia escamosa o endocervical) y el segundo grupo formado por 83 pacientes con frotis diagnosticado como Neoplasia Intraepitelial grado I. Todas las pacientes seleccionadas tenían diagnóstico histopatológico de Cervicitis crónica en biopsia incisional, posterior al informe de citología.
\end{abstract}

RESULTADOS: Durante el seguimiento en el primer grupo no se observó progresión de la lesión. En el segundo grupo se diagnosticó Lesión Intraepitelial de alto grado en dos pacientes $(2,40 \%)$ y cáncer invasor en dos pacientes $(2,40 \%)$.

CONCLUSION: En seguimiento a mediano plazo la tendencia general de las alteraciones atípicas cervicales es hacia la regresión. En los casos en que se observó progresión ésta se presentó después de los 36 meses.

PALABRAS CLAVES: Atipia escamosa, atipia endocervical, atipia epitelial de significado indeterminado.

SUMMARY

OBJECTIVE: This study determine the risk of developing intraepithelial neoplasm or invasive cancer in patients with epithelial atypia in cervical smears.

METHOD: This research compare 135 patients, divided into two groups, which were studied during 36 months. The first group was composed by 52 women with atypical cells of undeterminated significance; the second group was composed by 83 patients which smears showed Low-grade squamous intraepithelial lesion (CIN I). All of the selected patients had histopathological diagnosis of chronic cervicitis by biopsy after citologycal report.

RESULTS: The first group did not showed progression of the lesion during the time of research, while in the second group two patients showed High-grade squamous intraepithelial lesion $(2.4 \%)$ and other two developed invasive cancer $(2.4 \%)$.

CONCLUSIONS: This study suggest that atypical cervical lesions have the tendency to heal. The cases that showed progression appeared after 36 months.

KEY WORDS: Atypical squamous cells, atypical endocervical cells, atypical cells of indeterminated significance.

\section{Introducción}

La incidencia de cáncer cervical en el Departamento del Cauca es un grave problema de salud pública.

En el Departamento de Patología se revisan anualmente un promedio de 22.000 citologías de las cuales aproximadamente el $6.8 \%$ son clasificadas como atipias benignas, el $0.08 \%$ como CIN I, el $0.02 \%$ CIN II. y el $0.2 \%$ CIN III. Se detectan un promedio de 74 casos de

* Médica Patóloga. Prof. Asociado. Depto. de Patología. Facultad de Ciencias de la Salud. Universidad del Cauca.

** Médica Patóloga. Prof. Asociado. Depto. de Patología. Facultad de Ciencias de la Salud. Universidad del Cauca.

*** Médico Epidemiólogo. Prof. Titular. Depto. de Medicina Familiar. Facultad Ciencias de la Salud. Universidad del Cauca. cáncer invasor por año contra 44 casos de Lesión intraepitelial de alto grado.

El análisis de nuestras estadísticas, desde 1975 a la fecha, muestra una tendencia ascendente del cáncer cervical invasor y cada vez las mujeres afectadas pertenecen a grupos etáreos más jóvenes.

El manejo de la paciente con atipia epitelial cervical es uno de los campos más controversiales en el área de la patología ginecológica porque aún no hay claridad en la definición del término "atipia" y esto se debe a que realmente no existe una delimitación neta entre las características morfológicas de un cambio inflamatorio severo y las de un proceso incipiente con potencial neoplásico del epitelio escamoso cervical. No solamente desde el punto de vista citológico sino también desde el punto de 
vista histológico $(27,32)$. El sistema Bethesda separa las formas de atipia de etiología incierta y las denomina células escamosas atípicas de significado indeterminado (ASCUS) ubicándolas en el mismo grupo de las neoplasias, lo cual no ha contribuido a aclarar los términos, pues continúa siendo oscura la interpretación de las lesiones ASCUS (27).

Algunos investigadores advierten que existe un significativo número de pacientes con PAP II y alguna forma de atipia que al ser evaluados por otros procedimientos diagnósticos tienen una neoplasia intraepitelial subyacente, cuya frecuencia podría llegar a ser del 20 al $48 \%$. El otro planteamiento se refiere a que la atipia puede progresar a grados significativos de displasia. Pero realmente hasta el momento no se ha podido precisar si las alteraciones atípicas progresan a displasia o simplemente son concomitantes. Además, al parecer, el comportamiento de la atipia escamosa estaría influido por la edad, después de los 40 años sería mucho menor el riesgo para desarrollar displasia. (2, 5-6, 12, 14-15, 17-21, 23, 26).

El presente es un trabajo de búsqueda y seguimiento a mediano plazo, de un grupo de pacientes con diagnóstico citológico de atipia epitelial de significado indeterminado (atipia escamosa o endocervical) su comportamiento clínico fue comparado con el de otro grupo de pacientes con citología clasificada como Lesión intraepitelial de bajo grado tipo CIN.I. En el estudio se observa que en seguimiento a mediano plazo la tendencia general de las alteraciones atípicas es hacia la regresión. La progresión, diagnosticada en un bajo porcentaje, se presentó 36 meses después del diagnóstico.

\section{Materiales y métodos}

Se seleccionaron para análisis 354 especímenes de biopsia cervical diagnosticados como cervicitis crónica, de pacientes con frotis citológico previo clasificados como atipia epitelial de significado indeterminado o Lesión intraepitelial de bajo grado tipo NIC I. Las citologías fueron revisadas por dos patólogos. El estudio se realizó entre enero de 1986 y diciembre de 1994, en el Hospital Universitario San José de Popayán.

Las biopsias y frotis citológicos se clasificaron según el Sistema Bethesda, utilizando parámetros citológicos e histológicos establecidos $(5,7,10,12,19,32)$.

Una vez clasificadas la biopsia y citología respectiva, se realizó el estudio de acuerdo a un protocolo de seguimiento, en el que se consignó la edad de la paciente, el número y grado de los controles citológicos realizados y la fecha en que fueron tomados: Se investigaron factores de riesgo, historia de infecciones cervicovaginales previas al reporte de atipia y el tipo de tratamiento recibido posterior a este diagnóstico.

El instrumento se aplicó directamente a la paciente y se estudió la historia clínica. Las pacientes que no tuvieron control citológico en el último año de seguimiento fueron localizadas, la mayoría de ellas residían en el medio rural; la toma de la citología se efectuó en sus respectivos puestos de salud y el frotis fue remitido para ser revisado por el grupo de trabajo. Cuando el resultado fue anormal, la paciente se remitió al Hospital Susana
López de Valencia para evaluación colposcópica, tomar biopsia dirigida y para programar su tratamiento de acuerdo al tipo de lesión.

Se excluyeron las pacientes con historia previa de anormalidad citológica, pacientes embarazadas al momento del diagnostico, pacientes con menos de un año de seguimiento, las biopsias que se motivaron por causas diferentes a las ya mencionadas, los diagnósticos de cervicitis crónica en histerectomías y conizaciones y los casos en los que la citología reportó coilocitos.

Se organizaron las pacientes en grupos, según el tipo de atipia y los años de seguimiento. Para el análisis estadístico de las variables se emplearon métodos de tendencia central y proporciones. Se utilizó el programa EPIINFO.

\section{Resultados}

Se seleccionaron 354 casos. De los cuales se pudo hacer seguimiento, un tiempo promedio de 36 meses, a 135 pacientes.

Las pacientes se clasificaron según el tipo de atipia, en dos grupos: En el primer grupo de 52 mujeres, se incluyeron todas las pacientes que tuvieron una citología con diagnóstico de Atipia escamosa de significado indeterminado (ASCUS) o atipia endocervical y el segundo grupo de 83 mujeres, formado por pacientes con citología clasificada como Lesión intraepitelial de bajo grado (LIBG) tipo CIN I. En el primer grupo, en el control citológico del primer año se detectó persistencia de la alteración en una paciente. En los años siguientes todos los controles citológicos fueron negativos, excepto en el octavo año, en el que se reportó un caso de recurrencia de los cambios atípicos. La persistencia o recurrencia de la atipia se observó en el $3.8 \%$ de los casos. En ningún caso se observó progresión de la enfermedad. Tabla 1. En el grupo de pacientes con LIBG tipo CIN I, en el primero y segundo año no se observó evolución a lesiones más severas, aunque hubo persistencia de discariosis en 2 casos (2.4\%). En los controles citológicos posteriores en el $2.4 \%$ se hizo diagnóstico de lesión intraepitelial de alto grado (LIAG) y en el $2.4 \%$ de cáncer invasor. Tabla 2 .

En estos casos los controles citológicos se corroboraron con biopsia. Ver estudio comparativo en la Tabla 3.

La investigación de los factores de riesgo fue muy difícil debido a las características socioculturales de las pacientes incluidas en el estudio. Dichos factores son comunes a los dos grupos por lo cual se analizan en conjunto.

Se observó que las alteraciones atípicas se presentan en mujeres jóvenes en edad fértil, la mayoría se encuentran entre los 20 y los 39 años. Tabla 4. Encontramos significativo que ninguna paciente menor de 33 años presentó persistencia o evolución a lesiones más severas, el mismo comportamiento se observó en pacientes mayores de 47 años. En tanto que todas las pacientes que tuvieron persistencia, recidiva o progresión se encontraron entre los 33 y 46 años. Tabla 5.

La edad de iniciación de relaciones sexuales es muy temprana, el promedio de edad fue de 19 con una mediana de 18 años. 
En 87 pacientes se puede establecer el número de compañeros sexuales, el $84 \%$ refiere un sólo compañero sexual.

En 66 casos fue posible investigar comportamiento del compañero sexual, el $65 \%$ admite que su compañero sexual es promiscuo, en una de estas pacientes se hizo diagnóstico de cáncer invasor en el tercer año de segui-

Tabla 1

\section{EVOLUCION DE PACIENTES CON ATIPIA EPITELIAL DE SIGNIFICADO INDETERMINADO}

\begin{tabular}{|ccccc|}
\hline $\begin{array}{c}\text { Años de } \\
\text { seguimiento }\end{array}$ & $\begin{array}{c}\text { No. de } \\
\text { mujeres }\end{array}$ & $\begin{array}{c}\text { Persisten/ } \\
\text { recurrencia }\end{array}$ & *LIAG & Cáncer \\
\hline 1 & 17 & 1 & - & - \\
2 & 10 & - & - & - \\
3 & 8 & - & - & - \\
4 & 4 & - & - & - \\
5 & 5 & - & - & - \\
6 & 3 & - & - & - \\
7 & 1 & - & - & - \\
8 & 4 & 1 & - & - \\
\hline Total & 52 & 2 & 0 & 0 \\
\hline
\end{tabular}

* LIAG: Lesión intraepitelial de alto grado.

Tiempo promedio de seguimiento: $3.5 \mathrm{~A}$

Persistencia/Recurrencia: $3.8 \%$

Progresión: 0\%

\section{FUENTE:}

Archivo Departamento de Patología, Fac. Ciencias de la Salud.

Archivo Hospital Universitario San José de Popayán.

Programa Patología Cervical Servicio de Salud del Cauca.

Tabla 2

EVOLUCION DE PACIENTES CON LESION INTRAEPITELIAL BAJO GRADO (CIN I)

\begin{tabular}{|ccccc|}
\hline $\begin{array}{l}\text { Años de } \\
\text { seguimiento }\end{array}$ & $\begin{array}{c}\text { No. de } \\
\text { mujeres }\end{array}$ & $\begin{array}{c}\text { Persisten/ } \\
\text { recurrencia }\end{array}$ & *LIAG & Cáncer \\
\hline 1 & 18 & 1 & - & - \\
2 & 18 & 1 & - & - \\
3 & 6 & - & 1 & 1 \\
4 & 9 & - & - & - \\
5 & 10 & - & - & 1 \\
6 & 8 & - & - & - \\
7 & 9 & - & - & - \\
8 & 5 & - & 1 & - \\
\hline Total & 83 & 2 & 2 & 2 \\
\hline
\end{tabular}

Tiempo promedio de seguimiento: $3.5 \mathrm{~A}$

Recurrencia: $2.4 \%$

Progresión:

\section{* Lesión intraepitelial de alto grado $2.4 \%$ Cáncer $2.4 \%$}

FUENTE:

Archivo Departamento de Patología Fac. Ciencias de la Salud UC.

Archivo Hospital Universitario San José de Popayán.

Programa Patología Cervical Servicio de Salud del Cauca.
Tabla 3

ESTUDIO COMPARATIVO DEL SEGUIMIENTO

\begin{tabular}{|ccccc|}
\hline Grupos & $\begin{array}{c}\text { No. de } \\
\text { mujeres }\end{array}$ & Recurrencia & *LIAG Cáncer \\
\hline *No. 1 & 52 & 2 & - & - \\
*No. 2 & 83 & 2 & 2 & 2 \\
\hline
\end{tabular}

*LIAG: Lesión intraepitelial de alto grado

*Grupo No. 1: Atipia epitelial de significado indeterminado.

*Grupo No. 2: Lesión intraepitelial de bajo grado (CIN I)

\section{FUENTE:}

Archivo Departamento de Patología. Fac. Ciencias de la Salud. Archivo Hospital Universitario San José.

Programa Patología Cervical Servicio de Salud del Cauca.

Tabla 4

RELACION ENTRE LA EDAD Y EL DIAGNOSTICO DE ATIPIA

\begin{tabular}{|c|c|c|c|}
\hline Tipo de Atipia & 33 años & 33 a 47 años & $>47$ años \\
\hline *ASCUS & 21 & 27 & 4 \\
\hline CIN I38 & 34 & 11 & \\
\hline Total pacientes & 59 & 61 & 15 \\
\hline
\end{tabular}

*ASCUS: Atipia epitelial de significado indeterminado La atipia se observa con frecuencia máxima entre las mujeres jóvenes y durante la edad reproductiva, posteriormente el descenso de la frecuencia es notable.

\section{FUENTE:}

Archivo Departamento de Patología. Fac. Ciencias de la Salud. Archivo Hospital Universitario San José.

Programa Patología Cervical Servicio de Salud del Cauca.

\section{Tabla 5}

\section{RELACION EDAD/EVOLUCION DE LA LESION} PROMEDIO DE SEGUIMIENTO 3 Y $1 / 2$ AÑOS

\begin{tabular}{|lccccc|}
\hline $\begin{array}{c}\text { Diagnóstico } \\
\text { post } \\
\text { seguimiento }\end{array}$ & $\begin{array}{c}<33 \text { años } \\
\mathrm{n}=59\end{array}$ & $\begin{array}{c}33 \text { a } 47 \text { a } \\
\mathrm{n}=61\end{array}$ & $\begin{array}{c}>48 \text { años } \\
\mathrm{n}=16\end{array}$ & Total \\
\hline *ASCUS & - & 2 & - & 2 \\
*LIBG & - & 2 & - & 2 \\
*LIAG & - & 2 & - & 2 \\
Cáncer & - & 2 & - & 2 \\
\hline Total & 0 & 8 & 0 & 8 \\
\hline
\end{tabular}

*ASCUS: Atipia epitelial de significado indeterminado

*LIBG: Lesión intraepitelial de alto grado

*LIAG: Lesión intraepitelial de bajo grado

La persistencia y/o recurrencia de las alteraciones atípicas al igual que la progresión a lesiones más severas se observó únicamente en el grupo etáreo de 33 a 47 años.

FUENTE:

Archivo Departamento de Patología. Fac. Ciencias de la Salud Archivo Hospital Universitario San José.

Programa Patología Cervical Servicio de Salud del Cauca. 
miento. En las pacientes cuyo compañero sexual no es promiscuo, hubo regresión de las alteraciones citológicas.

En 84 pacientes se pudo investigar antecedentes de infecciones cérvico-vaginales previas al diagnóstico, observamos que el $62 \%$ tuvo al menos un episodio de infección, causado por gérmenes como tricomonas, hongos, Gardnerella Vaginales, Gonococos y hubo un caso de sífilis. Fue en este grupo donde se observó el más alto índice de progresión a lesiones epiteliales más severas y cáncer. En 25 casos no se dio ningún tipo de tratamiento para la infección, en éstas últimas el seguimiento no evidenció alteraciones.

De las 135 pacientes incluidas en el estudio, 77 pacientes recibieron crioterapia posterior al diagnóstico de atipia, 20 pacientes no recibieron ningún tipo de tratamiento y no se obtuvo información de 38 pacientes. Entre las pacientes que no recibieron crioterapia una progresó a LIAG.

\section{Discusión}

En el seguimiento a mediano plazo de un grupo de pacientes con Atipia epitelial de significado indeterminado (grupo No. 1) se observó que el patrón de comportamiento usual es la regresión y en un bajo porcentaje se presenta recurrencia.

Monz et al. de la Universidad de California hacen seguimiento, sin tratamiento, durante 9 meses a un grupo de pacientes con atipia escamosa y reportaron regresión de la lesión en el $78.3 \%$ de los casos (22); en tanto que lo observado en el presente estudio fue una regresión del $97 \%$.

En el grupo No. 2 no hay concordancia entre el informe citológico y el resultado de la biopsia. La citología de estas pacientes demostró una LIBG (NIC I) pero tienen, al igual que en el grupo anterior, diagnóstico de cervicitis crónica en la biopsia. En este grupo en el $4.8 \%$ se observó progresión de la enfermedad, la cual se evidenció 36 meses después del diagnóstico. Este hallazgo es semejante al observado por M.H. Jones et als. en un seguimiento a mediano plazo a 214 pacientes con discariosis leve, quienes fueron controladas con citologías periódicas y no se les administró tratamiento (13).

En el presente estudio observamos que el riesgo para desarrollar Lesión Intraepitelial de Alto grado o cáncer, es mayor en pacientes cuya citología muestra discariosis leve, aunque su biopsia sea negativa. Por esto, cuando las alteraciones observadas en la citología son más severas que las reportadas por la biopsia y existe una diferencia de por lo menos un grado, la paciente debe ser remitida a colposcopia y biopsia, e incluso si persiste el diagnóstico negativo debe recurrirse al cono diagnóstico. Esto con el fin de detectar alteraciones más severas. En este caso, la citología correctamente interpretada, debe ser una guía confiable en la búsqueda de una lesión que no se hace evidente en la biopsia $(4,8 ; 10,17,22,25,30)$.

Con respecto a los factores de riesgo encontramos que las alteraciones atípicas se presentan en mujeres jóvenes de edad fértil, la mayoría se encuentra entre los 20 y los 39 años (29).

En los dos grupos estudiados la edad parece ser un factor de riesgo importante en la evolución de las lesiones intraepiteliales. Ninguna paciente menor de 33 años o mayor de 47 años presentó evolución a las lesiones más severas; en tanto que las pacientes que si lo hicieron se encontraron entre los 33 y los 47 años. Lo referido concuerda con lo reportado por Hirschowitz en un estudio de 437 pacientes, quien ubica el riesgo de progresión entre los 29 y los 40 años (11).

Otros estudios amplían el rango a edades más avanzadas $(15,20)$. Con base en lo anterior la edad podría ser un indicador sensible del riesgo en las pacientes con atipia citológica no clasificada como CIN.

Hay que señalar que el $100 \%$ de las pacientes incluidas en el estudio tenían factores de riesgo. El 79\% había iniciado actividad sexual en edad muy temprana, el estrato socioeconómico de más del $95 \%$ de las pacientes es bajo, y se observó una elevada frecuencia de infecciones cervicovaginales recidivantes $(3,5,24,29,31)$.

La edad de iniciación de actividad sexual está relacionada con la aparición temprana de alteraciones atípicas citológicas y con el riesgo para desarrollar cáncer (29).

La mayoría de las pacientes refiere un sólo compañero sexual. El factor promiscuidad, ya sea de la mujer o de su compañero, fue significativo, en este grupo una paciente tuvo diagnóstico de cáncer invasor durante el seguimiento $(5,21)$.

En las pacientes con antecedentes de infecciones cervicovaginales previas al diagnóstico, encontramos el más alto índice de riesgo, en todas las mujeres en quienes durante el seguimiento se diagnosticó: recurrencia, persistencia, Lesión intraepitelial de alto grado o Cáncer invasor, estuvo presente este factor. Según los resultados del presente estudio, la historia de infecciones cervicovaginales parecería ser el indicador más sensible del riesgo, ya que ellas indirectamente no están hablando de la persistencia de múltiples factores, como la promiscuidad por ejemplo, la cual puede inferirse a través de su diagnóstico.

Con base en el comportamiento de la atipia epitelial de significado indeterminado observado en este estudio, creemos que el tratamiento ideal debe incluir tres elementos: criocauterio, control citológico anual en tanto persista el riesgo y educación en salud a la pareja para minimizar los riesgos y evitar que la paciente se pierda. Consideramos que no es necesario someterla a tratamientos más agresivos, de un alto costo y que conllevan un trauma sicológico innegable para la paciente y su familia.

En las pacientes que tengan factores de riesgo con el tratamiento adecuado y controles citológicos anuales regulares, si su alteración patológica progresa, ésta será detectada antes de que llegue a convertirse en un cáncer invasor. Según el Dr. Richart, el riesgo para desarrollar cáncer en pacientes con CIN de cualquier grado, tratadas con crioterapia, no es mayor que el que podría tener una mujer en la población general (28).

A diferencia de lo planteado por investigadores ingleses, quienes recomiendan estricto seguimiento a las pacientes con discariosis leve o moderada, pero no tratamiento inmediato $(1,11,13,16)$, en nuestro medio, en donde no tenemos forma de tipificar infección HPV concomitante, y por lo tanto no podemos determinar el riesgo real de las pacientes (27), consideramos que es 
necesario dar tratamiento a estas lesiones, apoyamos lo anterior, anotando además que cada paciente tiene un potencial biológico individual que hasta el momento no es susceptible de medición y que no nos permite predecir quienes van a evolucionar a cáncer (9).

En nuestro país el problema real de esta enfermedad no está en el manejo de las pacientes que regularmente asisten a su control citológico, el problema es el de las mujeres que nunca han tenido un control citológico y es a éste grupo al cual se debe canalizar todo el esfuerzo para educar y proteger, ya que en la forma en que están planteadas las cosas, está demostrado que el cáncer de cérvix es una enfermedad transmisible, por lo tanto la educación sanitaria sobre este aspecto es uno de los parámetros más importantes para lograr su control.

Proponemos que se cree un programa en donde se brinde educación, soporte sicológico sobre su problema y un control citológico anual idóneamente procesado e interpretado, con lo cual disminuiremos en forma muy significativa los.costos en el manejo de las pacientes con atipia epitelial, los cuales se deben invertir en la ampliación de la cobertura a la población femenina de alto riesgo.

\section{Agradecimientos}

Los autores expresan su agradecimiento a:

Martha Cecilia Vásquez, Enfermera Mg. Profesora de la Facultad de Enfermería, Universidad del Valle, Asesora en la parte metodológica; Angel Ceballos M., MD. Patólogo, Profesor de la Facultad de Ciencias de la Salud, Universidad del Cauca; Otto Citelly, MD. Jefe Programa Materno Infantil, Salud Pública; Lelia Eugenia Cabanillas, Citóloga del Programa de Patología Cervical, Hospital Susana López Valencia, Servicio de Salud del Cauca; María Piedad Acosta, MD. Ginecóloga, Profesora de la Facultad de Ciencias de la Salud, Universidad del Cauca y Jefe del Programa de Patología Cervical. Servicio de Salud del Cauca; José Vicente Eraso, MD. Ginecólogo, Profesor de la Facultad de Ciencias de la Salud; Médicos Rurales y Enfermeras Jefes del Programa de Patología Cervical. Servicio de Salud del Cauca; Profesores del Departamento de Patología. Facultad de Ciencias de la Salud. Universidad del Cauca; Programa PRODEN Universidad del Cauca - Universidad del Valle; Hospital Universitario San José de Popayán; Servicio de Salud del Cauca.

\section{BIBLIOGRAFIA}

1. Anderson David, MD., Flannelly Grainne MD., Mild and Moderate dyskariosys: can women be select for colposcopy on the basis of social criteria? Br Med J 1992; 305: 84-86.

2. Averette H. MD., What Management for atypical Pap Smear? Symposium. Contemp Obs/Gyn 1989; 237-245.

3. Barton S., Maddox P et al. Possible Cofactors in the Etiology of Cervical Intraepithelial Neoplasia. Un inmunopathologic Study. J. Reprod. Med. 1989; 34: 613-616.

4. Benedet JL. MD., Anderson GH., MD. A Comprehensive Programa for Cervical Detection and Management. Am. J. Obstet. Gynecol. 1992; 166: 1254-1259.

5. Blaustein A., ed. Pathology of the Female Genital Tract. 3rd ed. New York: Springer-Verlag 1989; 158-165.

6. Busseniers Ana E., Sidawy Mary. Inflamatory Atypia on Cervical Smears. J. Reprod. Med. 1991; 36: 85-88.

7. Davos G., Hernández Eet als. Atypical Squamous Cells in Papanicolau Smears. Obstet. Gynecol. 1987; 69: 43-46.

8. Gall Stanley MD., Pap Smears. Postgrad Med. 1989; 85: 235-239.

9. Helson and Albores Savedra. Pathology of Incipient Neoplasia. Mayor Problems in Pathology. Series Editor 1992; 248.

10. Himmelstein L., Evaluation of inflammatory Atypia. A Literature Review. J. Reprod. Med. 1989; 34: 634-636.

11. Hirschowitz Lynn, MD et als. Long term follow up of women with borderline cervical smear test results: effects of age and viral infection on progression to high grade dyskaryosis. Br Med J 1992; 304: 1209-1212.

12. Jones DE, MD., Creasman W et als. Evaluation of the atypical Pap Smear. Am. J. Obstet. Gynecol. 1987; 157: 544-549.

13. Jones MH, MD., Jenkins D. MD et als. Mild cervical dyskaryosis: safety of cytological surveillance. Lancet 1992; 339: 1440-1443.

14. Kambour A., Mauser $\mathrm{N}$ et als. MD. Inflamatory Atypia in Cervicovaginal Smears. Acta Cytol 1988; 32: 756-757.

15. Kaminski P., Stevens C., Wheelock J. Squamous Atypia on Cytology. The influence of Age. J. Reprod. Med. 1989; 34: 617-620.

16. Kirby AJ, MD., Spiegelhalter DJ et als. Conservative treatment of mild/moderate cervical dyskaryosis: long-term outcome. Lancet 1992; 339: 828-831.

17. Koss Leopold, MD. The Papanicolau Test for Cervical Cancer Detection. A Triumph and a Tragedy. Jama 1989; 261: 737-743.
18. Lawley $\mathrm{T}$., Lee R et als. The Significance of Moderate and Severe Inflamation on Class I Papanicolau Smear. Obstet. Gynecol. 1990; 76: 997-999.

19. Macord M., Stovall Summitt et al. Discrepancy of Cervical Cytology and Colposcopic Biopsy: Is Cervical Conization Necessary? Obstet Gynecol 1991; 77: 715-719.

20. Symmans F. MD., Mechanic L. MD et als. Correlation of Cervical Cytology and Human Papilomavirus DNA Detection in Postmenopausal Women. Int-J-Gynecol-Pathol 1992; 11: 204-209.

21. Miller K., Losh D et als. Evaluation and Follow-up of Abnormal Pap Smears. Am Fam Physician 1992; 45: 143-149.

22. Montz FJ. MD., Monk BJ, MD et als. Natural History of the Minimal Abnormal Papanicolau Smear. Obstet. Gynecol. 1992; 80: 385-387.

23. Noumoff J, MD. Atypia in Cervical Cytology as a Risk for Intraepithelial Neoplasia. Am. J. Obstet. Gynecol. 1987; 156: 628-631.

24. Pearlstone A. MD., Grigsby P. MD., Mutch D. MD. High Rates of Atypical Cervical Cytology: Occurrence and Clinical Significance. Obstet. Gynecol. 1992; 80: 191-195.

25. Piver S, MD. Controversia sobre el Papanicolau. Mundo Médico julio-1990; 33-44.

26. Reiter Robert, MD. Management of Initial Atypical Cervical Cytology: A Randomized, Prospective Study. Obstet. Gynecol. 1986; 68: 237-240.

27. Richart Ralph, MD et als. Managing patients with low-grade Pap smear. Cover story-Symposium. Contemp Obs/Gyn 1993; 82-94.

28. Richart Ralph MD., Townsend D. MD et als. An analysis of "longterm" follow results in patients with cervical intraepithelial neoplasia treated by cryotherapy. Am. J. Obstet. Gynecol. 1980; 137: 823-826.

29. Robbins SL. Patología Estructural y Funcional 3ra. ed. Editorial Panamericana; págs. 1198-1202.

30. Rosai Juan MD., Ackerman Surgical Pathology Vol. 2. 7th ed. Ed. Mosby 1989; 1040-1042.

31. Smith PG, MD., Kinlen LJ et als. Mortality of Wives of Mens Dying with Cancer of the penis. Br. J. Cancer 1980; 41: 422-428.

32. Tabbara S. MD., Saleh AB. MD et als. The Bethesda Classification for Squamous Intraepithelial Lesions: Histologic, Cytologic, and Viral Correlates. Obstet. Gynecol. 1992; 79: 338-346. 